\title{
The expression of marker genes during the differentiation of mesenchymal stromal cells
}

\author{
Aleksandra Zołocińska ${ }^{\mathrm{A}-\mathrm{F}}$ \\ Department of Regenerative Medicine, Maria Skłodowska-Curie Memorial Cancer Center, Warszawa, Poland \\ A - research concept and design; B - collection and/or assembly of data; $C$ - data analysis and interpretation; \\ $D$ - writing the article; $E$ - critical revision of the article; $F$ - final approval of the article
}

Address for correspondence

Aleksandra Zołocińska

E-mail: aleksandradebska88@gmail.com

Funding sources

The work was supported by OPUS UM0-

2013/11/B/ST8/03401, National Science Center.

Conflict of interest

None declared

Received on August 23, 2016

Reviewed on January 2, 2017

Accepted on January 10, 2017

\begin{abstract}
Mesenchymal stromal cells (MSCS) are an excellent and easily accessible source of precursor cells that have applications in regenerative medicine. They can be obtained from almost any tissue; however, bone marrow, Wharton's jelly and adipose tissue are the most frequently used sources of MSCs. Increased interest in using MSC in medical procedures has resulted in a pressing need to identify the genetic elements that can indicate the presence and the characteristics of MSCs. Genomic profiling enables the identification and characterization of MSCs as well as finding biomarkers and key molecules involved in all processes occurring in the cell. This knowledge is essential for developing a stem cell approach for tissue engineering and can improve the development of new clinical applications of MSCs. This review is an attempt to give an overview of key genetic markers indicating the main directions of MSC differentiation. The expression of these genes provides information about the direction and progress of differentiation and about interactions with the surrounding environment as well as specific molecular pathways that MSCs are involved in.
\end{abstract}

Key words: gene expression, mesenchymal stem cells, regenerative medicine, genetic markers

DOI

10.17219/acem/68386

\section{Copyright}

Copyright by Author(s)

This is an article distributed under the terms of the

Creative Commons Attribution Non-Commercial License

(http://creativecommons.org/licenses/by-nc-nd/4.0/) 


\section{Introduction}

Mesenchymal stem cells (MSCs) are multipotent progenitor cells that are capable of differentiating into osteogenic, chondrogenic, and adipogenic lineages at least (Fig. 1). ${ }^{1}$ It has been noted that MSCs may demonstrate plasticity beyond their traditional mesodermal lineage and that under specific conditions they can differentiate into neural cells, cardiomyocytes, pancreatic islet beta-cells, and hepatocytes. ${ }^{2-5}$ According to the definition of the International Society for Cellular Therapy (ISCT), MSCs are plastic-adherent cells, expressing a panel of surface markers while lacking expression of hematopoietic-related antigens. ${ }^{1}$ Their phenotype is characterized by a synthesis of surface markers:

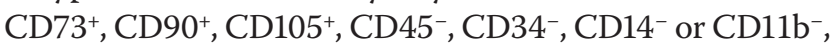
and $\mathrm{CD} 79 \mathrm{a}^{-}$or $\mathrm{CD}^{-} 9^{-}$. Knowledge of these surface markers is crucial for their identification, characteristics and isolation by sorting. ${ }^{6}$ Initially, MSCs were identified in mice bone marrow aspirates, the spleen and the thymus, but further studies have revealed the presence of MSCs in most organs of the adult body as well as in cord blood, Wharton's jelly, the perivascular area, and under the amniotic membrane of the umbilical cord, the placenta, and the amniotic fluid. ${ }^{6,7}$ However, the only MSCs with a practical application are cord blood MSCs, Wharton's jelly MSCs (WJ-MSCs), bone marrow stromal cells (BM-MSCs) and adipose-derived stromal cells (ASCs). In particular, a number of studies have reported that the isolation of stromal cells from adipose tissue is endowed with a high proliferative capacity and differentiation potential; ASCs are also easily isolated from lipoaspirate. ${ }^{8}$

Mesenchymal stromal cells are an easily accessible source of precursor cells that can find applications in regenerative medicine, improving myocardial function, therapies of Crohn's disease, and neurodegenerative disorders. ${ }^{9}$ Adipose-derived stromal cells are also used for adipose tissue regeneration in patients following breast cancer surgery, ${ }^{10}$ the acceleration of skin wound healing, regenerative treatment of widespread traumatic calvarial bones defects, the repair of tracheomediastinal fistulas caused by cancer ablation, the treatment of chronic ulcers caused by radiation therapy and controlling graft-versus-host disease (GVHD), and the treatment of osteoarthritis (OA). ${ }^{9,10}$

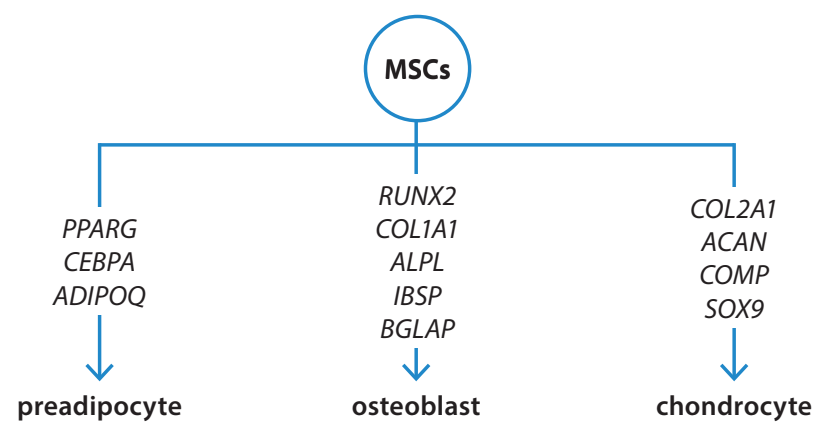

Fig. 1. Scheme of genes activated during MSCs differentiation into adipogenic, osteogenic, and chondrogenic lineages
The main function of MSCs is considered to be generating connective tissue and forming the stromal cells for bone marrow. Recent studies also revealed that MSCs are able to modulate the functions of the immune system. Taking into account the growing interest in using MSCs in medical procedures, there is a pressing need to identify the genetic elements that can indicate the presence of MSCs and their differentiation into various tissues. Numerous studies have examined the genomic profiles of MSCs, identifying potential biomarkers and key molecules that regulate biological processes involved in cell growth, development and survival. The knowledge gained from these studies may help in the development of strategies of improving the tissue regeneration potential of MSCs for various clinical applications. ${ }^{11}$

There are several methods that enable the detection of the directions of MSCs differentiation. The simplest method is staining differentiated cells using specific dyes. Mesenchymal stromal cells differentiated into osteogenic lineage can be detected by alizarin red staining, which detects the formation of extracellular matrix calcification. A differentiation toward an adipocyte phenotype can be assessed by Oil Red O staining in order to visualize the presence of lipid droplets. The acquisition of a chondrogenic phenotype can be evaluated with the use of alcian blue. Staining, however, is not reliable enough to be the only method that determines the directions of differentiation: it has to be confirmed by more specific techniques like real-time polymerase chain reaction (RT-PCR), immunocytochemistry, or the comparison of miRNA expression profiles. ${ }^{12}$ Immunocytochemistry is the most common method of detecting MSCs in the examined tissue, because it enables an analysis of the presence and location of any antigens in the cell and the identification of particular cell types by combining the fluorophore-labeled antibody with a suitable surface antigen. This method, however, has some disadvantages, such as the possibility of false positive or negative results as well as non-specific fluorescence. Besides, direct immunocytochemical methods have a poor sensitivity and a high cost, while in turn, an indirect method procedure is very complex and long; they also have higher risk of non-specific binding of secondary antibody resulting in background staining. ${ }^{13}$

Very precise methods include the comparative genomewide gene expression profile analysis and microRNA expression profiling. It is known that these small non-coding RNA molecules' occurrence is specific to cell type and that they modulate gene expression and/or protein synthesis, but little is known about their global expression profiles in mesenchymal stromal cells. Therefore, mRNA expression data, when combined with genome-wide gene expression profiles, can provide a great deal of information about transcriptional networks and differentiation processes in MSCs. ${ }^{14}$ RNA-seq, using NGS (next generation sequencing) to indicate the presence and quantity of RNA as well as cDNA microarrays, are other very accurate methods of assessing the gene expression level; however, they are 
expensive and their results require difficult data analysis. In comparison to these methods, real-time PCR seems to be the most appropriate way to define the differentiating potential of MSCs through measuring the expression of marker genes specific for particular tissue.

\section{Quantitative assessment of the expression of marker genes}

Real-time PCR technology enables the determination of the cell differentiation stage by gene expression monitoring, which allows us to detect an active differentiation mechanism very early, depending on the chosen marker genes. Quantitative real-time PCR is a high-throughput, sensitive and fast method that can measure the increase in DNA copy numbers in real time. ${ }^{15}$ For the detection of the number of cDNA copies in tested samples, researchers use molecules that emit light after binding to double-stranded DNA, e.g., SYBR Green or ethidium bromide. However, using primers and SYBR Green dye may result in binding non-specific sequences, which gives false results because SYBR Green emits light when bound to any double-stranded DNA.

A more specific and reliable method involves the use of fluorescent probes, e.g., TaqMan ${ }^{\text {TM }}$ probes, which are labeled with 2 different fluorescent dyes: a reporter dye at the $5^{\prime}$ and a quenching dye at the 3 '. In order to generate light emission, the reporter dye molecule must be separated from the quencher dye molecule. Primers and probes sequences are available in multiple publications; they can also be designed using specialized software, based on sequences in the National Center for Biotechnology Information (NCBI) or the Ensembl database. Real-time PCR is an integral method, widely used in molecular diagnostics. This technique is extremely sensitive and allows for simultaneous expression analysis of several samples. In addition, the results generated by the software and obtained from different experiments can be combined and compared with each other. However, this technique has some disadvantages, as the accuracy of the results depends largely on the probes and endogenous control used. The choice of a proper internal control gene is essential for obtaining reliable results, because a critical factor for creating reliable data in relative quantification is the normalization of the expression data of the genes of interest. This selection is particularly important when the expression differences are slight or when the samples derive from different histological origins or stages of development. Reference genes are called "house-keeping genes" and their expression in tissue is stable, irrespective of the conditions. ${ }^{16}$

Progenitor cells undergo a series of stable identity transitions on their way to becoming fully differentiated cells with unique identities. The gene expression profile determines the mode of cell differentiation; it is the most fundamental level at which a genotype gives rise to a phenotype.
At the beginning of the gene expression, transcription factors initiate transcription and RNA polymerase binds to the promoter sequence in order to synthesize pre-mRNA. Due to post-transcriptional processes, mature mRNA is formed and transported from the nucleus to the ribosomes in a cytoplasm, where translation begins and the information encoded in mRNA is translated to an amino acid chain.

Using quantitative real-time PCR, the differentiation potential of MSCs can be confirmed very early, at every step of gene expression before the formation of protein, producing results for several studied genes at once very fast and easily.

\section{Differentiation marker genes}

During cell differentiation, certain genes specific to the tissue are expressed (Table 1). To determine if MSCs differentiate into a particular tissue, a few differentiationrelated genes have to be chosen. Molecular basis and transcription factors activated during the differentiation of particular tissues of mesodermal origin have been broadly described. While examining the differentiation of MSCs, the expression of genes involved in the direction of differentiation is checked. Gene expression analysis can be performed using primers or probes for selected genes or prepared gene expression arrays for specific biological processes, e.g., $\mathrm{RT}^{2}$ Profiler ${ }^{\mathrm{TM}}$ PCR Array, with assays for differentiation-associated genes and assays for endogenous control genes. The second option is easy and it does not require the selection of appropriate marker genes or the matching of endogenous controls, but the cost is very high and 3-5 marker genes are sufficient to check whether there is a differentiation process. Designing primers or probes, however, is associated with a risk, because the accuracy of results depends on the quality of the used primers or probes and the endogenous control. ${ }^{17}$

Table 1. List of markers of MSCs differentiation into osteogenic, adipogenic, and chondrogenic lineages

\begin{tabular}{|c|c|c|}
\hline $\begin{array}{l}\text { Direction of MSCs } \\
\text { differentiation }\end{array}$ & $\begin{array}{l}\text { Activated } \\
\text { genes }\end{array}$ & References \\
\hline Chondrogenic & $\begin{array}{l}\text { COL2A1 } \\
\text { ACAN } \\
\text { COMP } \\
\text { SOX9 }\end{array}$ & $\begin{array}{l}\text { Hamid et al. }{ }^{32} \\
\text { de Crombrugghe et al. }{ }^{33} \\
\text { Lin et al. }{ }^{34} \\
\text { Mwale et al. }{ }^{35}\end{array}$ \\
\hline Adipogenic & $\begin{array}{l}\text { PPARG } \\
\text { CEBPA } \\
\text { ADIPOQ }\end{array}$ & $\begin{array}{l}\text { Graneli et al. } .^{19} \\
\text { Ntambi et al. }{ }^{20} \\
\text { Hu et al. }{ }^{21}\end{array}$ \\
\hline Osteogenic & $\begin{array}{l}\text { RUNX2 } \\
\text { COL1A1 } \\
\text { ALPL } \\
\text { IBSP } \\
\text { BGLAP }\end{array}$ & $\begin{array}{l}\text { Graneli et al..11 } \\
\text { Qi et al. }{ }^{23} \\
\text { Marom et al. }{ }^{25} \\
\text { Mizuno et al.. } \\
\text { Tsai et al. }{ }^{27} \\
\text { Eid et al. } .^{28} \\
\text { Gordon et al. }{ }^{29} \\
\text { Maes et al. }{ }^{31}\end{array}$ \\
\hline
\end{tabular}

MSCs - mesenchymal stem cells. 


\section{Adipogenic differentiation}

Mesenchymal stromal cells differentiate in vitro at least toward adipogenic, osteogenic and chondrogenic lineages when treated with established lineage-specific factors. The selective differentiation of these cells depends on specific environmental cues, usually a combination of growth factors and cytokines, which are supplied in vitro. In order to differentiate MSCs toward the adipogenic lineage, cells must be cultured in fetal bovine serum (FBS) containing Dulbecco's modified Eagle's medium (DMEM), supplemented with dexamethasone, insulin, and isobutylmethylxanthine (IBMX) and/or indomethacin and thiazolidinedione (TZD). ${ }^{12,18}$

One of the most commonly used marker genes in the detection of adipogenic differentiation of MSCs is PPARG (peroxisome proliferator-activated receptor gamma). The PPARG gamma protein, encoded by this gene, is a transcription factor, the main switch in adipogenic differentiation of MSCs, and has been implicated in the pathology of such diseases as obesity, diabetes, atherosclerosis and cancer. It is reported that the expression of the PPARG gene has a negative effect on osteogenic differentiation and that its inhibition of PPAR- $\gamma$ during the induction of osteogenesis leads to increased osteogenic differentiation of human MSCs. ${ }^{19}$ The expression of the $P P A R-\gamma$ gene is transcriptionally induced within 2 days following the induction of differentiation and lasts until day 3 to 4 at the latest. The $P P A R-\gamma$ expression is mediated by the activity of $C / E B P \beta$ and $\delta$, which also mediate the expression of $C / E B P \alpha$ - another marker gene of adipogenic differentiation. ${ }^{20} C / E B P \beta$ and $\delta$ are the first transcription factors induced after preadipocyte exposure to a differentiation medium, as their expression starts increasing a few hours after induction. Once the induction medium is removed from the culture, the expression of $C / E B P \delta$ dissipates over the subsequent $48 \mathrm{~h}$, whereas the decline of $C / E B P \beta$ is more gradual, with its expression approximating $0 \%$ of maximal levels by day 8 . The expression of $C / E B P \alpha$ increases from undetectable levels in preadipocytes to detectable levels 2 days after stimulation with adipogenesisspecific factors, and to full expression 5 days after differentiation is initiated. The genes of the $C / E B P$ family are involved in directing the process of differentiation during adipogenesis and, together with $P P A R-\gamma$, are required for full adipocyte differentiation. ${ }^{20}$ Therefore, the $C / E B P$ family genes and $P P A R-\gamma$ are markers of early adipogenesis. One late adipogenesis marker gene is $A D I P O Q$, whose expression is highly specific to adipose tissue and is observed exclusively in mature fat cells as the stromal-vascular fraction of fat tissue does not contain $A D I P O Q s$ mRNA. The expression of $A D I P O Q s$ mRNA is a late event in adipogenesis, first appearing on approx. day 4 after the induction of differentiation. ${ }^{21}$

\section{Osteogenic differentiation}

The most promising area for MSCs application is bone reconstruction and regeneration. Osteogenesis is a complex process that involves the differentiation of mesenchymal cells into pre-osteoblasts and osteoblasts, which ultimately leads to the synthesis and deposition of bone matrix proteins. ${ }^{22}$ In order to differentiate mesenchymal stromal cells toward the osteogenic lineage, cells need to be cultured in osteogenic medium, containing dexamethasone, ascorbate-2-phosphate, $\beta$-glycerophosphate, l-glutamine, ascorbic acid, and/or vitamin $\mathrm{D}_{3} \cdot{ }^{12,22}$

Bone formation is known to occur through the activation of molecules as the runt-related transcription factor 2 (RUNX2), type I collagen (COL1A1), alkaline phosphatase (ALPL), integrin-binding sialoprotein (IBSP), and bone gamma-carboxyglutamate (gla) protein (BGLAP), which orchestrate the differentiation of MSCs into functional osteoblasts. The most important transcription factor in osteogenesis is RUNX2, which plays a crucial role in the formation of the mineralized skeleton during embryogenesis and regulates the maturation of the osteoblast phenotype. ${ }^{23,24}$ It is crucial for the formation of mineralized tissue and its expression is usually associated with the early phases of osteogenic differentiation. ${ }^{11}$ RUNX2 is the main regulator of the osteogenic differentiation of MSCs and it regulates the transcription of osteocalcin (BGLAP). Osteocalcin is a matrix protein that regulates osteoclast activity; it is highly specific for mineralization and is a good marker of the osteoblastic phenotype. ${ }^{25,26}$ The BGLAP gene encodes a protein associated with the mineralized bone matrix, secreted by the calcified tissue and regulated by vitamin $\mathrm{D}_{3}$; it indicates the activity of osteoblasts and it is known as a marker of late osteogenesis.

Another gene which is activated by RUNX2 is COL1A1. Collagen I is the pervading protein of the bone matrix, and its expression and secretion is crucial to the mineralization process. Attachment to collagen I is mediated through integrins that activate kinase signaling pathways, thus supporting osteoblast cell proliferation and bone growth. ${ }^{25}$ COL $1 A 1$ is essential for osteoblast adhesion and bone growth. ${ }^{26}$ Furthermore, type I collagen is the main constituent of the organic part of the extracellular matrix (ECM) ${ }^{11}$ The next most frequently used marker of osteogenic differentiation, alkaline phosphatase $(A L P)$, is the effector protein responsible for the mineralization of the extracellular matrix. ${ }^{11}$ Alkaline phospatase is an early bone marker protein and an essential enzyme for ossification. As $A L P$ is widely used as a marker of osteoblasts, an increase in its activity should be associated with osteoblastic differentiation. When the mineralization process is well progressed, the $A L P$ level increases and then decreases; the peak of its expression falls on day 7 after induction. ${ }^{27}$ $A L P$ is closely related to pre-osseous cellular metabolism and to the elaboration of calcified bone matrix. ${ }^{28}$

Integrin-binding sialoprotein (IBSP) is an acidic, noncollagenous glycoprotein abundantly expressed in mineralized tissues and a major structural protein of the bone matrix. ${ }^{29}$ This protein binds to calcium and hydroxyapatite through its acidic amino acid clusters and mediates cell 
binding through an arginylglycylaspartic acid (RGD) sequence, which recognizes the vitronectin receptor. ${ }^{30}$

Generally, IBSP and BGLAP are late markers of osteogenesis, as are osteopontin (OPN) and msh homeobox 2 (MSX2), whereas RUNX2, COL1A1 and ALP are markers of early osteogenesis, along with the Sp7 transcription factor (OSX) and transforming growth factor-beta 1 (TGFB1). ${ }^{22-31}$

\section{Chondrogenic differentiation}

Cartilage defects caused by trauma, tumor ablation or age-related abrasion lead to constant pain and functional limitations, causing a decreased quality of life. It is known that even small lesions can affect the structure and function of the articular cartilage, predisposing it to the development of osteoarthritis (OA). The reason for this is a lack of vascularization and enervation in the articular cartilage tissue, which inhibits repair processes like inflammation and fibrin clot formation. Only chondrocytes and synoviocytes residing in the local environment can fill up the defects by slow proliferation and matrix deposition. ${ }^{31}$ Troubles caused by the nature of cartilage tissue have inspired further studies, which focus on the possibilities of regeneration of this tissue. Mesenchymal stromal cells are one of the most promising research areas. Chondrogenic differentiation requires a high density cell culture and monolayer culture reduces the ability of differentiation towards chondrogenic lineage; therefore, the best solution is 3-dimensional culture created using scaffolds made of natural or synthetic materials. The chondrogenic induction medium is enriched with glutamax (Invitrogen Carlsbad, USA), vitamin C, insulin-transferrin-selenium$\mathrm{X}$ (ITS), insulin-like growth factor 1 (IGF-1), ascorbate2-phosphate, L-proline, TGF- $\beta$, and dexamethasone. ${ }^{32}$

At the beginning of chondrogenesis, MSCs aggregate and condensate, while expressing various extracellular matrix components, including type II collagen (COL2A1), aggrecan core protein (ACAN), cartilage oligomeric protein (COMP), and SRY-box 9 (SOX9). After differentiating into chondrocytes, the cells begin to produce ECM materials rich in aggrecan core protein and cartilage oligomeric protein, forming cartilage tissue. ${ }^{32}$

Type II collagen is an early marker of chondrogenesis, expressing in chondroprogenitor cells and in chondrocytes ${ }^{33}$; the expression of the COL $2 A 1$ gene was reduced at the $2^{\text {nd }}$ and $3^{\text {rd }}$ week of the culture. ${ }^{32}$ The SOX9 expression, however, reached the highest level at the $3^{\text {rd }}$ week of culture, promoting a production of cartilage matrix. This protein also expresses in chondroprogenitor cells and chondrocytes, but there is no $S O X 9$ expression in hypertrophic chondrocytes. ${ }^{33}$ Lin et al. confirms that the expression of COL2A1 and SOX9 is an early and unique marker of chondrogenesis. ${ }^{34}$ Another early marker of chondrogenesis is COMP, whose expression reaches the highest level 1 week after chondrogenic induction. ${ }^{32}$ In contrast, aggrecan and type X collagen are markers of late chondrogenesis, related to chondrocyte hypertrophy. ${ }^{35}$
Chondrogenesis in mesenchymal stem cells is most prominent after 1 week of chondrogenic induction, and longer stimulation can induce chondrocyte hypertrophy. ${ }^{32}$

\section{Other differentiation directions}

Mesenchymal stromal cells seem to be an easily accessible source of precursor cells that can be expanded in vitro and used for many clinical applications. As mentioned above, MSCs may demonstrate plasticity beyond their traditional mesodermal lineage. Under specific conditions, they are able to differentiate into mesodermal lineage cells (adipocytes, chondrocytes, osteoblasts, tenocytes, or myocytes), endodermal lineage cells (astrocytes or neurons) or ectodermal lineage cells (hepatocytes or islet beta cells). ${ }^{3-6}$ There is a need to create a panel of markers for each direction of MSC differentiation. The expression of MYOD1 (myogenic differentiation 1), MYF5 (myogenic factor 5) and MYOG (myogenin) transcription factors is crucial for myogenic differentiation and a high expression of TNC (tenascin C), SCX (scleraxis bHLH transcription factor), $D C N$ (decorin), and COL1A2 (collagen type I alpha 2 chain) genes occurs during tenogenic differentiation of MSCs. ${ }^{6,36}$ During neurogenic differentiation of MSCs, there is an expression of genes related to nervous system development: nestin, used to identify neural stem and early progenitor cells, GFAP - a structural element of fibrillary astrocytes - and NeuN, a neuronal nuclear antigen. ${ }^{2}$ Mesenchymal stromal cells induced to differentiate into a islet beta cell phenotype express genes which encode hormones specific for the pancreas: ISL1 (insulin), NGN3 (an early pancreatic progenitor marker), SST (somatostatin), and GCG (glucagon) - a specific endocrine marker. ${ }^{4}$ Differentiation into hepatocytes results in the expression of AFP (alphafetoprotein) and $H N F 1 \beta$ (hepatocyte nuclear factor $1 \beta){ }^{5}$

\section{Reference genes}

Gene expression analyses are essential to the discovery and characterization of the roles for known genes and to understanding the processes occurring in cells. Regarding the study of the development of different tissues, gene expression analyses can provide insights into complex regulatory networks that coordinate in cells such processes as proliferation, cell commitment, differentiation, and apoptosis. Genes which exhibit constant expression levels among all tissues regardless of conditions are reference genes. The perfect reference gene should have similar expression levels regardless of experimental conditions, mainly developmental stages, composition of cell types and sample treatments.

The most commonly used reference genes are $\beta$-actin and GAPDH; however, recent studies have shown that these common reference genes are not stably expressed under all experimental conditions. Ideally, reference genes 
should show stable expression levels in all studied cell types and tissues, and transcription should be constant relative to the general cellular transcription rates under different experimental conditions.

Nonetheless, research has proven that the expression of individual reference genes varies among samples under different experimental conditions. Therefore, it is relevant to select a proper reference gene for normalization, according to experimental settings. In recent years, it has been found that gene expression should be normalized using at least 3 reference genes to ensure that the experiment results are reliable. ${ }^{37}$

\section{Summary}

Tissue engineering offers potential methods of repairing damaged tissues, and it is a rapidly growing field of regenerative medicine. The ability to differentiate in multiple directions, the immunomodulation properties and the regulation of endogenous tissue repair make MSCs very attractive for regenerative medicine. In recent years, the use of adipose-derived stromal cells have particularly gained popularity. Adipose tissue can be described as an easily available source of stromal vascular fraction (SVF), which contains preadipocytes, endothelial progenitor cells, T cells, B cells, mast cells, adipose tissue macrophages, and MSCs. In 2001, Zuk et al. demonstrated that adiposederived stromal cells are capable of differentiating into adipo-, chondro-, osteo-, and myogenic lineages. ${ }^{38}$ A year later, the same authors proved that ASCs express the same surface markers as bone marrow: derived mesenchymal stromal cells (BM-MSCs): CD29, CD44, CD71, CD90, and CD105, lacking the expression of CD31, CD34, and CD45. ${ }^{39}$

Real-time PCR enables the definition of the common genetic profile of BM-MSCs and ASCs, and the examination of a complex network of molecules which regulate the homing and communication of MSCs with their environment by activating signaling pathways that play a crucial role in maintaining their stemness properties. Saulnier et al. identified 190 cohort modulated transcripts, which may be molecular MSCs' stemness signature. Among them, there were genes involved in basic biological mechanisms like embryogenesis, signal transduction, cell adhesion, and inter-cellular communication. ${ }^{8}$

The biggest advantage of ASCs over BM-MSCs lies in the collection of a much larger number of cells with less of the risk associated with surgical procedure. Cells derived from lipoaspirate are also a more homogenic population than those derived from bone marrow, which makes ASC applications more efficient than the use of BM-MSCs. ASCs as well as BM-MSCs have already been used for cellbased therapies, including orthopedic disorders, cardiovascular diseases, graft vs host disease, and Crohn's disease. ${ }^{3,9}$ The use of stem cells in medicine is growing, so there is a need for an accurate characterization of these cells.
Knowledge of rapid changes in gene expression during stem cell differentiation will provide a better understanding of the potential of these cells for regenerative medicine.

Numerous studies have examined genomic profiles of MSCs collected from different tissues, which differ in their ability to proliferate and differentiate into various lineages. Genomic profiling enables the characterization of MSCs and the identification of biomarkers and key molecules involved in the regulation of cell survival, growth and development. The knowledge gained from these studies will improve the understanding of basic processes occurring in the stem cell and may help in the development of strategies for improving the clinical applications of MSCs. ${ }^{2-10}$

Comprehensive knowledge of the biological processes involved in tissue repair is essential for developing a stem cell approach for tissue regeneration. Understanding their molecular regulation during the repair process is crucial in order to achieve the desired clinical outcomes. Overall, molecular studies on differentiating MSCs identify potential markers that can be used to isolate and purify MSCs from heterogeneous cell populations. They also provide data on potential genomic indicators involved in specific differentiation pathways that can disclose information about the stage of differentiation of the tested cells. Importantly, however, the detection of differentiation-related gene expression is insufficient for successful phenotypic differentiation. Further experiments are required in order to confirm the differentiation. ${ }^{11-16}$

In conclusion, gene expression analysis during MSCs differentiation provides information regarding the direction and progress of differentiation and exhibits the activation of specific molecular pathways MSCs are involved in as well as their interactions with the surrounding environment.

\section{References}

1. Horwitz EM, Le Blanc K, Dominici M, et al. Clarification of the nomenclature for MSC: The International Society for Cellular Therapy position statement. Cytotherapy. 2005;7(5):393-395.

2. Safford KM, Hicok KC, Safford SD, et al. Neurogenic differentiation of murine and human adipose-derived stroll cells. Biochem Biophys Res Commun. 2002;294:371-379.

3. Toma C, Pittenger MF, Cahill KS, Byrne BJ, Kessler PD. Human mesenchymal stem cells differentiate to a cardiomyocyte phenotype in the adult murine heart. Circulation. 2002;105:93-98.

4. Chen LB, Jiang XB, Yang L. Differentiation of rat marrow mesenchymal stem cells into pancreatic islet beta-cells. World J Gastroenterol. 2004;10:3016-3020.

5. Ji R, Zhang N, You N, et al. The differentiation of MSCs into functional hepatocyte-like cells in a liver biomatrix scaffold and their transplantation into liver-fibrotic mice. Biomaterials. 2012;33:8995-9008.

6. Pojda Z, Machaj E, Kurzyk A, et al. Mesenchymal stem cells. Postepy Biochem. 2013;59(2):187-197.

7. Friedenstein AJ, Gorskaja JF, Kulagina NN. Fibroblast precursors in normal and irradiated mouse hematopoetic organs. Exp Hematol. 1976;4:267-274.

8. Saulnier N, Puglisi MA, Lattanzi W, et al. Gene profiling of bone marrow- and adipose tissue-derived stromal cells: A key role of Kruppel-like factor 4 in cell fate regulation. Cytotherapy. 2011;13:329-340.

9. Mizuno H. Adipose-derived stem cells for tissue repair and regeneration: Ten years of research and literature review. J Nippon Med Sch. 2009;76(2):56-66. 
10. Włodarski K, Włodarski P, Galus R, Mazur S. Adipose mesenchymal stem cells. Their characteristics and potential application in tissue repair. Pol Orthop Traumatol. 2012;77:97-99.

11. Graneli C, Thorfve A, Ruetschi U, et al. Novel markers of osteogenic and adipogenic differentiation of human bone marrow stromal cells identified using a quantitative proteomics approach. Stem Cell Res. 2014;12:153-165.

12. Huang SJ, Fu RH, Shyu WC, et al. Adipose-derived stem cells: Isolation, characterization, and differentiation potential. Cell Transplant. 2013;22:701-709.

13. Luongo de Matos L, Trufelli DC, Luongo de Matos MG, da Silva Pinhal MA. Immunohistochemistry as an important tool in biomarkers detection and clinical practice. Biomark Insights. 2010;5:9-20.

14. Bae S, Ahn JH, Park CW, et al. Gene and microRNA expression signatures of human mesenchymal stromal cells in comparison to fibroblasts. Cell Tissue Res. 2009;335:565-573.

15. Heid CA, Stevens J, Livak KJ, Williams PM. Real time quantitative PCR. Genome Res. 1996;6:986-994.

16. Walder RY, Wattiez AS, White SR, de Prado BM, Hamity MV, Hammond DL. Validation of four reference genes for quantitative mRNA expression studies in a rat model of inflammatory injury. Mol Pain. 2014;10:55.

17. Wu L, Cai X, Zhang S, Karperien M, Lin Y. Regeneration of articular cartilage by adipose tissue derived mesenchymal stem cells: Perspectives from stem cell biology and molecular medicine. J Cell Physiol. 2013;228;938-944.

18. Taha MF, Hedayati V. Isolation, identification and multipotential differentiation of mouse adipose tissue-derived stem cells. Tissue Cell. 2010;42:211-216.

19. Graneli C, Karlsson C, Brisby H, Lindahl A, Thomsen P. The effects of PPAR $-\gamma$ inhibition on gene expression and the progression of induced osteogenic differentiation of human mesenchymal stem cells. Connect Tissue Res. 2014;55(4):262-274.

20. Ntambi JM, Kim YC. Adipocyte differentiation and gene expression. J Nutr. 2000;130:3122S-3126S.

21. Hu E, Liang P, Spiegelman BM. AdipoQ is a novel adipose-specific gene dysregulated in obesity. J Biol Chem. 1996;271(18):10697-10703.

22. Sila-Asna M, Bunyaratvej A, Maeda S, Kitaguchi H, Bunyaratvej N. Osteoblast differentiation and bone formation gene expression in strontium-inducing bone marrow mesenchymal stem cell. Kobe J Med Sci. 2007;53(1-2):25-35.

23. Qi H, Aguiar DJ, Williams SM, La Pean A, Pan W, Verfaillie CM. Identification of genes responsible for osteoblast differentiation from human mesodermal progenitor cells. Proc Natl Acad SciU SA. 2003;100(6);3305-3310.

24. Prince $M$, Banerjee $C$, Javed $A$, et al. Expression and regulation of Runx2/Cbfa1 and osteoblast phenotypic markers during the growth and differentiation of human osteoblasts. J Cell Biochem. 2001;80:424-440.

25. Marom R, Shur I, Solomon R, Benayahu D. Characterization of adhesion and differentiation markers of osteogenic marrow stromal cells. J Cell Physiol. 2005;202:41-48.

26. Mizuno M, Kuboki Y. Osteoblast-related gene expression of bone marrow cells during the osteoblastic differentiation induced by type I collagen. J Biochem. 2001;129:133-138.

27. Tsai MT, Li WJ, Tuan RS, Chang WH. Modulation of osteogenesis in human mesenchymal stem cells by specific pulsed electromagnetic field stimulation. J Orthop Res. 2009;27(9):1169-1174.

28. Eid AA, Hussein KA, Niu LN, et al. Effects of tricalcium silicate on osteogenic differentiation of human bone marrow-derived mesenchymal stem cells in vitro. Acta Biomater. 2014;10:3327-3334.

29. Gordon JAR, Tye CE, Sampaio AV, Underhill TM, Hunter GK, Goldberg HA. Bone sialoprotein expression enhances osteoblast differentiation and matrix mineralization in vitro. Bone. 2007;41:462-473.

30. http://www.uniprot.org/uniprot/P21815. Published May 1, 1991. Updated February 28, 2018. Accessed April 15, 2018.

31. Maes C, Kobayashi T, Selig MK, et al. Osteoblast precursors, but not mature osteoblasts, move into developing and fractured bones along with invading blood vessels. Dev Cell. 2010;19:329-344.

32. Hamid AA, Idrus RBH, Saim AB, Sathappan S, Chua KH. Characterization of human adipose-derived stem cells and expression of chondrogenic genes during induction of cartilage differentiation. Clinics. 2012;67(2);99-106.
33. de Crombrugghe B, Lefebvre V, Behringer RR, Bi W, Murakami S, Huang W. Transcriptional mechanisms of chondrocyte differentiation. Matrix Biol. 2000;19:389-394.

34. Lin Y, Luo E, Chen X, at al. Molecular and cellular characterization during chondrogenic differentiation of adipose tissue-derived stromal cells in vitro and cartilage formation in vivo. $J$ Cell Mol Med. 2005;9(4):929-939.

35. Mwale F, Stachura D, Roughley P, Antoniou J. Limitations of using aggrecan and type $X$ collagen as markers of chondrogenesis in mesenchymal stem cell differentiation. J Orthop Res. 2006;24:1791-1798.

36. Burk J, Gittel C, Heller S, at al. Gene expression of tendon markers in stromal cells derived from different sources. BMC Res Notes. 2014;7:826.

37. An Y, Reimers K, Allmeling C, Liu J, Lazaridis A, Vogt PM. Validation of differential gene expression in muscle engineered from rat groin adipose tissue by quantitative real-time PCR. Biochem Biophys Res Commun. 2012;421:736-742.

38. Zuk PA, Zhu M, Mizuno $H$, et al. Multilineage cells from human adipose tissue: Implications for cell-based therapies. Tissue Eng. 2001;7:211-226.

39. Zuk PA, Zhu M, Ashjian P, et al. Human adipose tissue is a source of multipotent stem cells. Mol Biol Cell. 2002;13:4279-4295. 